\section{Contise}

Correspondence to

Professor Timothy John Williams, National Heart and Lung Institute, Faculty of Medicine, Imperial College London, SAF Building, South Kensington, London SW72AZ, UK tim.williams@imperial.ac.uk

Received 25 June 2012 Accepted 7 August 2012

\title{
New cell for asthma: enter the myeloid
}

\author{
Timothy John Williams
}

The discovery of a new type of inflammatory cell ${ }^{1}$ would not feature in the wish list of a student of immunology grappling with the congested pathways of multiple cytokine molecules regulating a myriad of cell-cell interactions. However, the chest physician will be acutely aware that, with all our advanced knowledge of mechanisms underlying allergic asthma, there is clearly an unmet need for new therapy which will depend on further dissection of the cross-talk between immune and somatic cells in the lung. While the majority of patients with asthma respond to treatment with bronchodilator drugs and corticosteroids, others at the severe end of the spectrum are difficult to treat. ${ }^{2}$ These patients generate a substantial morbidity and socio-economic burden and asthma exacerbations carry a significant risk of fatal attacks.

The new cell type discovered by Petersen et al surfaced in the analysis of data from a model of chronic asthma in the mouse. Animal models, particularly in the mouse, have given us a working hypothesis that underpins the understanding of basic mechanisms of allergy. Data that build on, or deviate, from this provide new opportunities to develop novel therapy that may prove effective in difficult-to-treat asthma, where corticosteroid insensitivity is a particular problem. A major advance from studies of mouse models was the recognition of the polarisation of $\mathrm{CD} 4$ thymusderived lymphocytes into T-helper (Th) 1 cells specialised to help defend against, for example, intracellular viral infection and Th2 cells specialised to defend against extracellular pathogens with a critical role in the defence against helminth parasites. These cells are tightly controlled by T-regulatory cells. Failure of regulation of Th1 cells leads to autoimmune diseases, such as rheumatoid arthritis, multiple sclerosis and diabetes, whereas failure to regulate Th2 cells results in allergy, an inappropriate triggering of inflammatory mechanisms by otherwise innocuous agents in the environment. Th2 cells secrete the interleukins IL-4, IL-5 and IL-13. These cytokines induce the symptoms of allergic asthma. IL-4 and IL-13 help in switching on the production of allergen-specific IgE production by $B$ cells. On exposure to allergens, crosslinking of IgE bound to lung mast cells induces the release of acute mediators responsible for the immediate symptoms of bronchospasm and mucus hypersecretion. IL-4 and IL-13 have multiple additional effects, for example, they switch on the production of eotaxins and other chemokines mediating eosinophil recruitment into the lung and contribute to airway remodelling. IL-5 is involved in the production and release of eosinophils from the bone marrow and prolongs their survival once in the lung tissue.

These mechanisms are used to explain the basics of asthma characterised by Th2 lymphocyte activation and the appearance of large numbers of eosinophils in the lung. However, it is clear from clinical practice that there are several subtypes of asthma, although no general consensus on the precise classification. ${ }^{3}$ Of particular concern is severe steroid-insensitive asthma, with debilitating virus-induced exacerbations characterised by neutrophil accumulation in the lung. The discovery of a third type of CD4 T lymphocyte, the Th17 cell, threw some light on mechanisms underlying this diversity. These cells secrete IL-17A and IL-17F cytokines that, amongst other activities, stimulate the production of chemokines that recruit neutrophils. Th17 cells are thought to be important in autoimmune reactions, but they are also implicated in subtypes of asthma, particularly those with neutrophil accumulation in the lung. The original IL-17 (now known as IL-17A) was joined by other members of an IL-17 family of proteins. Another of the IL-17 family, IL-25 (IL-17E), is secreted by airway epithelial cells (and also other cells, such as mast cells and eosinophils) in response to inflammatory stimuli. IL-25 is known to amplify allergic reactions by stimulating Th2 cells to produce high levels of Th2 cytokines. ${ }^{4}$

Enter now a new type of cell, described by Petersen et al, that is a granulocyte derived from a myeloid precursor in the bone marrow, rather than a cell derived from a lymphoid precursor, but this cell secretes the Th2 lymphocyte cytokines, IL-4 and IL-13. These cells, type 2 myeloid (T2M) cells, express receptors for IL-25. Significantly, IL-4 and IL-13 secretion switched on by IL-25 in these cells is corticosteroid insensitive. Thus, these cells may well be relevant to severe, steroid-insensitive asthma patient phenotypes.

The secretion of Th2 cytokines by myeloid cells is in itself not new. The waters had already been muddied by several publications in the past showing that mast cells, basophils, eosinophils and M2 macrophages can produce IL-4 and IL-13. A pertinent question is why T2M cells have not been seen before in allergic reactions (although related cells may have been observed previously in response to a helminth parasite infection ${ }^{5}$ ). One answer may lie in the experimental protocol employed in the discovery. The authors sensitised mice with a cockroach allergen/adjuvant mixture and gave multiple allergen challenges to produce a severe, chronic airways disease. They found that the allergic response to challenge in the lung was associated with upregulation of IL-25 and the 
Figure 1 Proposed role of the type 2 myeloid (T2M) cell in the airway wall of a patient with severe asthma. IL, interleukin.

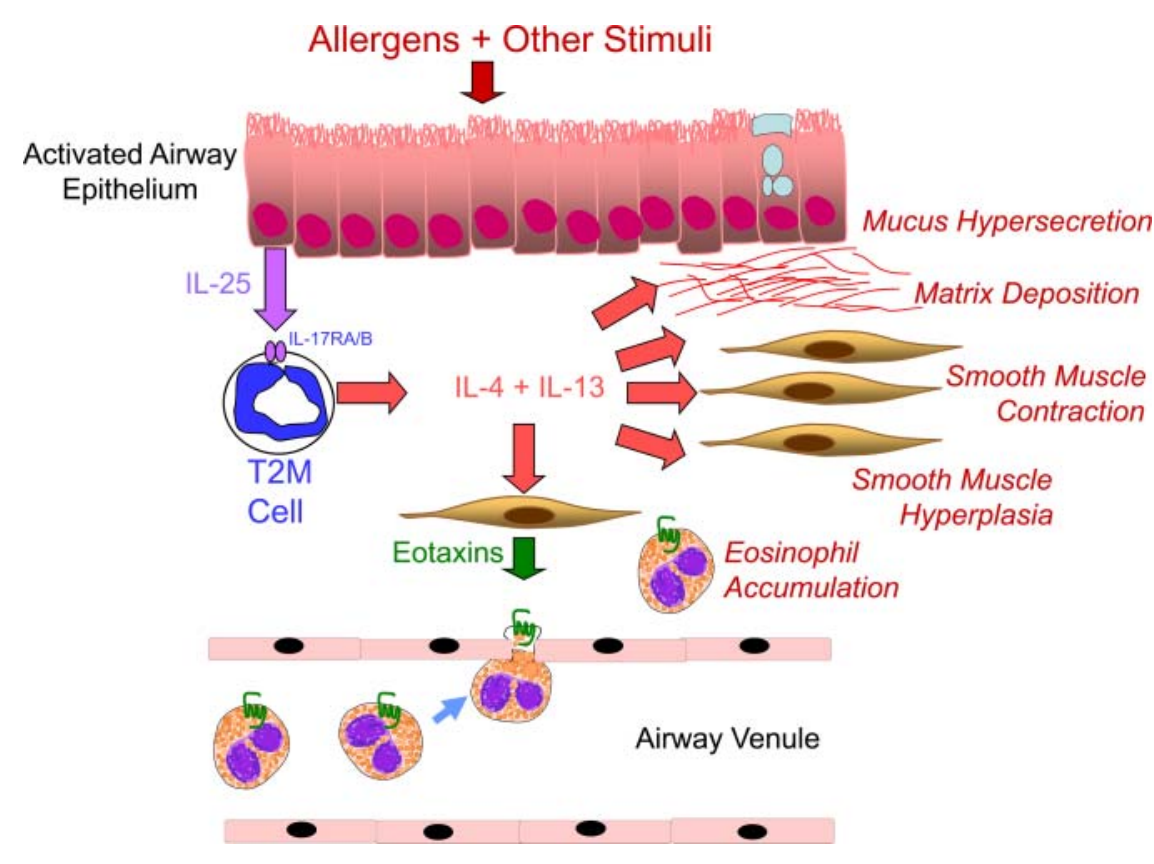

appearance of large numbers of myeloid cells expressing the IL-25 receptor. The T2M cells were far in excess of classic Th2 lymphocytes. Genetic modification to delete a component of the IL-25 receptor (IL-17RB) resulted in a reduction in numbers of T2M cells and Th2 lymphocytes. Administration of IL-25 into the airways of mice induced the appearance of T2M cells expressing IL-4 and IL-13. These cells were not observed when using cockroach antigen in more acute sensitisation protocols.

Petersen et al looked for evidence of T2M-like cells in human blood. They found a population of IL-17RB + cells bearing myeloid markers, but lacking the lymphocyte markers CD4 and CD8. The numbers of these cells were elevated in the circulation of patients with asthma. These cells produced IL-4 and IL-13 when stimulated with IL-25 in vitro. Again, the question arises why these cells have not been observed previously in man. From the profile of gene expression in mouse T2M cells the authors conclude that, amongst granulocytic cells, these cells are closest to eosinophils. A comparison was made between T2M, eosinophils, neutrophils and macrophages (mast cells and basophils were not included). The human T2M-like cells described by Petersen et al expressed markers found on neutrophils, such as CD16, and also a neutrophil/myeloid progenitor marker CD177. The morphology shown in the paper for T2M cells resembles that of band-type neutrophils. Could these cells have been mistaken for immature neutrophils in previous studies?

Thus, the boundaries between previously well defined cell types and their functions are becoming blurred by observations such as those in Petersen et al. This may prove an irritation for scholars, but possibly a life-line for those charged with seeking new therapeutic targets for patients with severe asthma whose disease profiles do not fit with the established paradigm (figure 1).

Competing interests None.

Provenance and peer review Commissioned; externally peer reviewed.

\section{REFERENCES}

1. Petersen BC, Budelsky AL, Baptist AP, et al. Interleukin-25 induces type 2 cytokine production in a steroid-resistant interleukin-17RB + myeloid population that exacerbates asthmatic pathology. Nat Med 2012;18:751-8.

2. Wang W, Li JJ, Foster PS, et al. Potential therapeutic targets for steroid-resistant asthma. Curr Drug Targets 2010;11:957-70.

3. Ogawa Y, Calhoun WJ. Phenotypic characterization of severe asthma. Curr Opin Pulm Med 2010;16:48-54.

4. Fort MM, Cheung J, Yen D, et al. IL-25 induces IL-4, IL-5, and IL-13 and Th2-associated pathologies in vivo. Immunity 2001;15:985-95.

5. Voehringer $\mathbf{D}$, Reese TA, Huang $X$, et al. Type 2 immunity is controlled by IL-4/L-13 expression in hematopoietic non-eosinophil cells of the innate immune system. J Exp Med 2006;203:1435-46. 